\title{
Avaliação da gramatura de cola na propriedade de flexão estática em painéis compensados de Copaifera duckei Dwayer e Eperua oleifera Ducke
}

\author{
Nerci Nina LIMA", Nabor da Silveira PIO²
}

\begin{abstract}
RESUMO
A presente pesquisa teve como objetivo analisar as seguintes gramaturas de cola: 270; 330; 364 e $390 \mathrm{~g} \cdot \mathrm{m}^{-2}$ a partir da produção de compensados fenólicos utilizando duas espécies florestais; Copaifera duckei e Eperua oleifera e avaliar a flexão estática dos painéis. A madeira foi proveniente da área de manejo florestal sustentado da Indústria de Madeira Compensada localizada em Manicoré, município do Amazonas. As lâminas foram produzidas na Gethal Amazonas S.A - Indústria de Madeira Compensada em Itacoatiara - AM com espessura de 2,2 mm. Foram produzidas a nível industrial 40 painéis, 20 por espécie no total de 8 tratamentos. O experimento foi completamente casualizado com arranjo fatorial. Foi utilizado a norma Européia EN 310: 1993. Os valores de densidade básica da espécie Copaifera duckei foi de 0,60 (g. $\left.\mathrm{cm}^{-2}\right)$ e 0,62 (g.cm-2) para Eperua oleifera. No ensaio de flexão estática, sentido paralelo à grã, somente a espécie Copaifera duckei apresentou diferença significativa a 5\% pelo Teste de Dunnette para as propriedades de MOE e MOR. No sentido perpendicular a grã, as espécies Copaifera duckei e Eperua oleifera tanto o MOE quanto o MOR apresentaram diferenças significativas a 5\% de probabilidade.
\end{abstract}

\section{PALAVRAS-CHAVE}

Gramatura de cola, Compensado, Flexão estática, Copaifera duckei, Eperua oleifera

\section{Evaluation of the glue amount in the static bending of the species Copaifera duckei Dwayer e Eperua oleifera Ducke}

\begin{abstract}
The objective of the present research was to analyze 270; 330; 364 and $390 \mathrm{~g} \cdot \mathrm{m}^{-2}$ amounts of glue in the production of fenolic plywood, using two forest species; Copaifera duckei and Eperua oleifera, and evaluate the static bending of the panels. The wood came from an area of sustained forest pertaining to the Plywood Industry located in Manicoré, county of Amazonas. The panels were produced with thicknesses of $2.2 \mathrm{~mm}$ at the Gethal Amazonas S.A in Itacoatiara - AM. Forty panels, 20 per species, were produced an industrial level, using a total of 8 treatments. The experiment was completely randomized with factorial arrangement. The European standard EN 310: 1993 was used. The basic density values were $0.60\left(\mathrm{~g} . \mathrm{cm}^{-2}\right)$ for Copaifera duckei, and $0.62\left(\mathrm{~g} \mathrm{~cm}^{-2}\right)$ for Eperua oleifera. In static bending, parallel to the grain, only Copaifera duckei presented a significant difference at 5\% in the Dunnette Test for the properties of MOE and MOR. In the perpendicular sense to the grain, Copaifera duckei and Eperua oleifera presented significant differences, for MOE as much as MOR, at 5\% of probability.
\end{abstract}

\section{KEYWORDS}

Glue amount, Plywood, Static bending, Copaifera duckei, Eperua oleifera.

\footnotetext{
1 Departamento de Ciências Florestais - UFAM, Av. Gal. Rodrigo Octávio Jordão Ramos 3000, Coroado, Manaus/AM. CEP 69077-000. Bloco A 221. e-mail: nlima@ufam.edu.br 2 Professor Adjunto. Dep. de Ciências Florestais da Universidade Federal do Amazonas - UFAM. Av. Gal. Rodrigo Octávio Jordão Ramos, 3000. Coroado. Manaus/AM. CEP 69077000. e-mail: nspio@ufam.edu.br
} 


\section{INTRODUÇÃO}

Como a floresta Amazônica é rica em diversidade madeireira, várias espécies apresentam resultados adequados para usos finais como móveis, construções leves e pesadas, embalagens de caixas, pallets, sobretudo na produção de chapas de compensado.

Pesquisas feitas atualmente são direcionadas a madeiras de reflorestamento como alternativa a utilização de madeiras tropicais por serem espécies de rápido crescimento e com grande potencial para laminação Iwakiri et al. (2002). Porém, poucos estudos são feitos com espécies tropicais provenientes da floresta amazônica como forma alternativa as espécies já tradicionalmente comercializada e em via de extinção.

A madeira por ser um material de origem orgânica responde de várias maneiras aos diversos tratamentos nela empregados, inclusive a aplicação de cola a qual depende do seu teor de umidade, tendendo a atingir um teor de umidade em equilíbrio dinâmico com a umidade relativa da atmosfera (Kollmann \& Cotê, 1968).

Um fator que influencia significativamente no processo de colagem e resistência da madeira é abaixa densidade e a alta permeabilidade (Marra, 1992).

$\mathrm{Na}$ colagem de lâminas de madeira para produção de compensados, devem-se levar em conta as características inerentes à madeira, o tipo a quantidade e a composição do adesivo, como também os procedimentos empregados na colagem, os quais têm importância fundamental na qualidade das chapas (Baldwin, 1975).

Para Sellers (1985), os fatores básicos que interferem no processo de colagem da madeira variam com a madeira, adesivo e condiçōes de colagem. Por isso a seleção do adesivo depende do uso final das chapas.

Utilização de lâminas finas com grande número de camadas caracteriza o compensado de uso estrutural/naval que são colados a base de resina fenol-formaldeído, conferindo ao mesmo alta resistência mecânica e resistência à umidade (Tsoumis, 1991).

O uso de laminação cruzada e restrição da linha de cola na formação do painel compensado estar em balancear os diferentes comportamentos físico-mecânicos das lâminas de camadas adjacentes, dispostas nos sentidos longitudinal e perpendicular ao plano da chapa (Bodig \& Jayne, 1982).

Suchsland (1972) cita que o balanceamento de um compensado com número ímpar de camadas pode ser alcançado, mesmo utilizando lâminas de espécies e espessuras diferentes, desde que o plano de simetria seja mantido, para o equilíbrio de parâmetros elásticos entre as lâminas que constituem o painel.

A influência da quantidade de cola na fabricação de compensados em geral é um fator importante, o que depende muito do teor de umidade o qual influi decisivamente na resistência da junta e na estabilidade do produto colado (Selbo,1975).

O estudo teve como objetivo avaliar por meio da flexão estática as diferentes gramaturas de cola na qualidade dos painéis compensados de Copaifera duckei e Eperua oleifera.

\section{MATERIAL E METÓDOS}

\section{COLETA DO MATERIAL}

As espécies utilizadas no presente estudo foram retiradas da área de manejo florestal sustentado da Indústria de Madeira Compensada Gethal Ltda localizada em Manicoré, município do Amazonas.

Após o abate, as toras foram transportadas por balsa para o município de Itacoatiara - AM, onde foi realizado todo o processo de laminação das toras na Gethal Amazonas S.A Indústria de Madeira Compensada.

Foram utilizadas duas espécies florestais: Copaifera duckei Dawyer, (mari-mari), família Caesalpiniaceae e Eperua oleifera Ducke, (copaíba jacaré), família Caesalpiniaceae, sendo utilizada 05 (cinco) árvores por espécie.

A madeira foi identificada no Laboratório de Anatomia da Madeira do CPPF- INPA.

\section{COZIMENTO DAS TORAS}

Após serem traçadas, receberam vaporização em tanques de cozimento a uma temperatura que variou entre $80^{\circ} \mathrm{C}$ a $100^{\circ}$ C, por um período de 72 horas, a fim de tornar a madeira mais plástica, melhorando seu processamento no torno.

Após o cozimento, as toras receberam um novo destopo para ficar nas dimensōes do torno desenrolador. As dimensōes seguiram o padrão da indústria em torno de $2,65 \mathrm{~m}$.

\section{FASES DE PRODUÇÃO DO COMPENSADO}

\section{PROCESSO DE LAMINAÇÃO}

O torno foi aferido de acordo com o padrão da empresa onde as lâminas foram produzidas com espessuras de 2,2 $\mathrm{mm}$.

\section{SECAGEM DAS LÂMINAS}

As lâminas foram secas em um secador industrial contínuo a uma temperatura de $120^{\circ} \mathrm{C}$ e tempo de secagem variando entre 25 e 30 minutos com teor de umidade médio final de $6 \%$.

\section{PRODUÇÃO DO COMPENSADO}

A manufatura dos compensados desde o cozimento até o esquadrejamento final, também foi realizada na Gethal Amazonas S.A - Indústria de Madeira Compensada. 
Depois de secas, as lâminas foram classificadas em duas categorias: a de melhor qualidade para capa; $\mathrm{e}$ as inferiores para miolo. Essa classificação foi baseada na Norma de Controle de Qualidade e Classificação de Compensados (IBDF, 1985).

Avaliou-se todo o processo do compensado dando ênfase no ciclo de prensagem otimizando a quantidade de cola usado para este fim

Quanto à umidade das lâminas, foram controladas através de medidores elétricos. $\mathrm{O}$ controle de espessura das lâminas foi acompanhado através de equipamento de precisão (micrômetro), onde foram realizadas medições aleatórias por espécie durante a laminação.

A resina utilizada na colagem das lâminas foi a fenolformaldeído. A composição do adesivo constituiu basicamente da mistura simples da resina em uma batedeira com as demais partes componentes da formulação adotada pela empresa conforme Tabela 1.

Tabela 1 - Formulação do adesivo fenol-formaldeídeo.

\begin{tabular}{llll}
\hline Adesivo & Componentes & Parte por peso & $(\%)$ \\
\hline \multirow{3}{*}{ Fenol-formaldeídeo } & Resina FF & 108 & 75,52 \\
\cline { 2 - 4 } & Farinha de trigo & 15 & 10,49 \\
\cline { 2 - 4 } & Água & 20 & 13,99 \\
\hline Total & & 143 & 100
\end{tabular}

O controle das propriedades do adesivo após seu preparo foi realizado através do copo Ford para leitura da viscosidade, e para a medição do $\mathrm{pH}$, verificando assim o estado e as condiçôes para utilização.

Foram utilizadas quatro diferentes gramaturas de cola aplicado em linha dupla: 270, 330, 364 e 390 g.m $\mathrm{m}^{-2}$, valores normalmente utilizados pelas indústrias de compensados, sendo a de 364 g.m-2 como testemunha. Foi utilizada meia batida o que equivale $108 \mathrm{~kg}$ de cola para as três gramaturas onde foram sendo ajustadas até a gramatura final.

Para a aplicação do adesivo foi calculada a quantidade de cola (gramatura), através da equação abaixo, e a aplicação do adesivo se deu através de aplicador de rolos industriais. Cada gramatura foi controlada através das aberturas entre o rolo aplicador e o rolo dosador, para assegurar uma distribuiç̧ão uniforme do adesivo.

$G r=\frac{P m-P s}{c . l}$

Em que:

$\mathrm{Gr}=$ gramatura em g.m-2

$\mathrm{Pm}=$ peso da lâmina molhado (cola) em gramas

Ps = peso da lâmina seco em gramas

$\mathrm{c}=$ comprimento da lâmina em metros
1 = largura da lâmina em metros

$\mathrm{O}$ intervalo entre um tratamento e outro variou de $10 \mathrm{a}$ 15 minutos e a montagem de cada painel teve em média de 4,6 minutos para espécie Copaifera duckei e 3,8 minutos para espécie Eperua oleifera, com o tempo total de assemblagem de cinco horas.

As variáveis do ciclo de prensagem seguiram as orientações técnicas estabelecidas pelo fabricante da resina, conforme Tabela 2.

Tabela 2 - Variáveis do ciclo de prensagem

\begin{tabular}{ll}
\hline Variáveis & Valores \\
\hline Adesivo & resina fenol-formaldeídeo \\
\hline Temperatura & $135^{\circ} \mathrm{C}$ \\
\hline Tempo de presnsagem & 20 minutos \\
Gramatura da cola & $270 ; 330 ; 364$ e $390 \mathrm{~g} / \mathrm{m}^{2}$ \\
\hline Pressão específica & $12 \mathrm{~kg} / \mathrm{cm}^{2}$ \\
\hline Umidade da lâmina & $6 \%$ \\
\hline
\end{tabular}

Cada chapa foi composta de nove lâminas com dimensões de $2,55 \times 1,22 \times 0,0022 \mathrm{~m}$, onde o número de chapas produzidas é mostrado no delineamento estatístico.

Após a elaboração dos painéis, os mesmos foram transportados e acondicionados na sala de climatização do Laboratório de Física da Madeira da Universidade Federal do Amazonas, a uma temperatura de $20^{\circ} \mathrm{C}$ até entrarem em equilíbrio com o ambiente e verificado aleatoriamente através de medidores elétricos.

Transcorrido esse tempo os mesmos foram transportados para a serraria do CPPF-INPA, onde foram confeccionados os corpos-de-prova para determinação da flexão estática no sentido paralelo e perpendicular ao comprimento dos corposde-prova.

\section{AVALIAÇÃO DOS PAINÉIS}

A principal variável a ser analisada foi a gramatura que variou nas seguintes quantidades: 270; 330; 364 e 390 g. $\mathrm{m}^{-2}$.

Quantos aos ensaios realizados e as normas utilizadas são mostrados na Tabela 3 .

Tabela 3 - Números de corpos de prova para ensaio por espécie e normas utilizadas

\begin{tabular}{|c|c|c|c|c|}
\hline Espécie & Ensaio & Condições & $\begin{array}{l}\text { Corpo de } \\
\text { prova }(p / p)\end{array}$ & Norma \\
\hline $\begin{array}{l}\text { Copaifera } \\
\text { duckei }\end{array}$ & $\begin{array}{l}\text { Flexão } \\
\text { estática }\end{array}$ & $\begin{array}{l}\text { paralelo } \\
\text { perpendicular }\end{array}$ & $\begin{array}{l}6 \\
6\end{array}$ & $\begin{array}{l}\text { EN 310: } \\
1993\end{array}$ \\
\hline $\begin{array}{l}\text { Eperua } \\
\text { oleifera }\end{array}$ & $\begin{array}{l}\text { Flexão } \\
\text { estática }\end{array}$ & $\begin{array}{l}\text { paralelo } \\
\text { perpendicular }\end{array}$ & $\begin{array}{l}6 \\
6\end{array}$ & $\begin{array}{l}\text { EN 310: } \\
1993\end{array}$ \\
\hline
\end{tabular}


A retirada dos corpos-de-prova dos painéis para o ensaio de propriedade mecânica é mostrada na Figura 1.

Figura 1 - Esquema das retiradas dos corpos de prova para o ensaio de flexão estática.

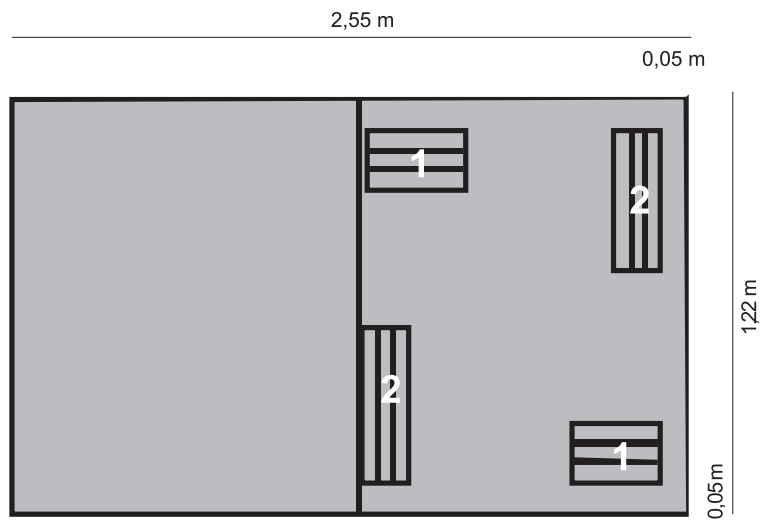

1- Flexão estática paralela $(450 \times 50 \mathrm{~mm})$

2- Flexão estática perpendicular $(450 \times 50 \mathrm{~mm})$

Fonte: Dados de pesquisa.

\section{ANÁLISE ESTATÍSTICA}

Após a realização dos ensaios mecânicos foi aplicado um delineamento inteiramente ao acaso, com arranjo em esquema fatorial dos tratamentos analisando o efeito da gramatura de cola em função das espécies na flexão estática como mostra a Tabela 4.

Tabela 4 - Espécies, Variáveis e Gramatura de cola aplicados nos tratamentos

\begin{tabular}{|c|c|c|c|c|}
\hline Espécies & Ensaios & Variáveis & Tratamentos & $\begin{array}{l}\text { Gramatura de } \\
\text { cola }\left(\mathrm{g} / \mathrm{m}^{2}\right)\end{array}$ \\
\hline \multirow{8}{*}{$\begin{array}{l}\text { Copaifera } \\
\text { duckei }\end{array}$} & \multirow{8}{*}{$\begin{array}{l}\text { Flexão } \\
\text { estática }\end{array}$} & \multirow{4}{*}{ Paralelo ao vão } & $\mathrm{T} 1$ & 270 \\
\hline & & & T2 & 330 \\
\hline & & & T3 & 364 \\
\hline & & & $\mathrm{T} 4$ & 390 \\
\hline & & \multirow{4}{*}{$\begin{array}{l}\text { Perpendicular } \\
\text { ao vão }\end{array}$} & $\mathrm{T1}$ & 270 \\
\hline & & & T2 & 330 \\
\hline & & & T3 & 364 \\
\hline & & & $\mathrm{T} 4$ & 390 \\
\hline \multirow{8}{*}{$\begin{array}{l}\text { Eperua } \\
\text { oleifera }\end{array}$} & \multirow{8}{*}{$\begin{array}{l}\text { Flexão } \\
\text { estática }\end{array}$} & \multirow{4}{*}{ Paralelo ao vão } & $\mathrm{T1}$ & 270 \\
\hline & & & T2 & 330 \\
\hline & & & T3 & 364 \\
\hline & & & $\mathrm{T} 4$ & 390 \\
\hline & & \multirow{4}{*}{$\begin{array}{l}\text { Perpendicular } \\
\text { ao vão }\end{array}$} & $\mathrm{T} 1$ & 270 \\
\hline & & & T2 & 330 \\
\hline & & & T3 & 364 \\
\hline & & & T4 & 390 \\
\hline
\end{tabular}

* Para cada tratamento foram efetuadas cinco repetições

A Tabela 5 mostra o esquema da análise de variância realizado.

Tabela 5 - Análise de Variância da interação gramatura x espécie

\begin{tabular}{ll}
\hline Fator de Variação & Graus de Liberdade \\
\hline Gramatura & 3 \\
Espécie & 1 \\
Gramatura x Espécie & 3 \\
Erro & 28 \\
Total & 39 \\
\hline
\end{tabular}

\section{RESULTADOS E DISCUSSÃO}

\section{FLEXÃO ESTÁTICA}

É um dos ensaios mais importantes para determinação das características mecânicas da madeira. Dois parâmetros são obtidos neste ensaio: o módulo de ruptura (MOR) e módulo de elasticidade (MOE).

Os valores médios de módulos de elasticidade (MOE) e módulo de ruptura (MOR) para o ensaio de flexão estática sentido paralelo e perpendicular ao comprimento dos corposde-prova das espécies estudadas estão apresentados na tabela 6 e 7, onde os resultados foram avaliados pela análise de variância e Teste de Dunnett, ao nível de 5\% de significância.

Tabela 6 - Valores médios de Flexão Estática sentido paralelo para os ensaios de módulo de elasticidade (MOE) e módulo de ruptura (MOR) para as espécies estudadas

\begin{tabular}{lllll}
\hline Espécie & Gramatura $\left(\mathrm{g} / \mathrm{m}^{2}\right)$ & T.U $(\%)$ & $\mathrm{MOE}\left(\mathrm{N} / \mathrm{mm}^{2}\right)$ & $\mathrm{MOR}\left(\mathrm{N} / \mathrm{mm}^{2}\right)$ \\
\hline \multirow{2}{*}{$\begin{array}{l}\text { Copaifera } \\
\text { duckei }\end{array}$} & T1 $(270)$ & 12 & $11.400,46^{\mathrm{ns}}$ & $68,02^{*}$ \\
& T2 (330) & 12 & $11.414,86^{\mathrm{ns}}$ & $78,35^{\mathrm{ns}}$ \\
& T3 (364) & 12 & $11.737,97^{\mathrm{ns}}$ & $83,44^{\mathrm{ns}}$ \\
& T4 (390) & 12 & $13.222,23^{*}$ & $85,53^{\mathrm{ns}}$ \\
\hline \multirow{2}{*}{$\begin{array}{l}\text { Eperua } \\
\text { oleifera }\end{array}$} & T1 (270) & 12 & $10.948,28^{\mathrm{ns}}$ & $71,66^{\mathrm{ns}}$ \\
& T2 (330) & 12 & $9.967,93^{\mathrm{ns}}$ & $69,57^{\mathrm{ns}}$ \\
& T4 (390) & 12 & $10.702,65^{\mathrm{ns}}$ & $75,50^{\mathrm{ns}}$ \\
\hline
\end{tabular}

*- Tratamento que diferem da testemunha ao nível de $5 \%$ pelo Teste de Dunnett

ns. Tratamento que não diferem da testemunha

Conforme o resultado da Tabela 6, observa-se que para o módulo de elasticidade e módulo de ruptura, a espécie Copaifera duckei, apresentou diferenças significativas em relação a testemunha, indicando dependência entre os fatores. Painéis produzidos com Copaifera duckei, (T4), $\mathrm{MOE}$, proporcionou valor médio superior à testemunha (T3), e o (T1), MOR, um valor médio inferior, ou seja, essas diferenças estão relacionados à gramatura, pois quanto maior a quantidade de cola maior é a resistência do MOE e MOR. 
Além disso, essa diferença pode estar relacionada com o pH dos extrativos presentes na madeira, principalmente o óleo encontrado na espécie que pode ter interferido com a reação de polimerização do adesivo, influenciando assim na formação e desempenho da ligação adesiva.

Para a espécie Eperua oleifera, tanto o módulo de elasticidade quanto o módulo de ruptura não apresentaram significância entre os valores médios, indicando que as diferentes gramaturas não influenciaram nestas propriedades podendo ser indicado a menor gramatura.

Quanto às espécies, verifica-se que há uma tendência de melhor desempenho para os painéis de Copaifera duckei em relação aos painéis de Eperua oleifera. Esse resultado, provavelmente pode estar relacionado a maior ou menor gramatura aplicada nos painéis independentemente entre as espécies ou a resistência mecânica da madeira.

No ensaio onde foram utilizadas amostras com a grã perpendicular ao comprimento dos corpos-de-prova os valores médios são apresentados na Tabela 7, onde apresentaram diferenças significativas.

Tabela 7 - Valores médios de Flexão Estática sentido perpendicular para os ensaios de módulo de elasticidade (MOE) e módulo de ruptura (MOR) para as espécies estudadas

\begin{tabular}{|c|c|c|c|c|}
\hline Espécie & Gramatura $\left(\mathrm{g} / \mathrm{m}^{2}\right)$ & T.U (\%) & MOE $\left(\mathrm{N} / \mathrm{mm}^{2}\right)$ & MOR $\left(\mathrm{N} / \mathrm{mm}^{2}\right)$ \\
\hline \multirow{4}{*}{$\begin{array}{l}\text { Copaifera } \\
\text { duckei }\end{array}$} & T1 (270) & 12 & $7.174,54^{\text {ns }}$ & $51,54^{*}$ \\
\hline & T2 (330) & 12 & $6.919,49^{\text {ns }}$ & $56,81^{\text {ns }}$ \\
\hline & T3 (364) & 12 & $7.264,25^{\text {ns }}$ & $60,95^{\text {ns }}$ \\
\hline & T4 (390) & 12 & $5.918,96^{\star}$ & $52,78^{\text {ns }}$ \\
\hline \multirow{4}{*}{$\begin{array}{l}\text { Eperua } \\
\text { oleifera }\end{array}$} & T1 (270) & 12 & $7.440,13^{\text {ns }}$ & $50,18^{\text {ns }}$ \\
\hline & T2 (330) & 12 & $8.480,52^{\star}$ & $64,53^{*}$ \\
\hline & T3 (364) & 12 & $6.484,62^{\mathrm{ns}}$ & $49,12^{\text {ns }}$ \\
\hline & T4 (390) & 12 & $7.874,79^{\text {ns }}$ & $57,43^{\text {ns }}$ \\
\hline
\end{tabular}

*- Tratamento que diferem da testemunha ao nível de $5 \%$ pelo Teste de Dunnett

ns. Tratamento que não diferem da testemunha

De acordo com a Tabela 7, observa-se que o valor médio do módulo de elasticidade espécie Copaifera duckei (T4), apresentou valor médio inferior em relação à testemunha como também para o módulo de ruptura em que o (T1) foi menor que a testemunha. Para as chapas de Eperua oleifera, tanto o módulo de elasticidade como o módulo de ruptura foi significativamente superior à testemunha no tratamento 2 . Da mesma forma, como no ensaio paralelo à grã dos corposde-prova essas diferenças estão relacionadas com a gramatura pois quanto maior a quantidade de cola nos limites testados maior é a resistência e rigidez do MOE e MOR.

Observa-se ainda que o MOE e o MOR dos painéis no sentido paralelo à grã do comprimento dos corpos-deprova apresentaram valores superiores em relação ao sentido perpendicular, evidenciando a influência da maior resistência da madeira no sentido paralelo à grã, pois Pereyra (1994) encontrou diferenças muito menores entre as duas direçōes ensaiando $E$. dunnil, $17 \%$ para o MOE e $12 \%$ para o MOR com painéis de nove camadas como do presente estudo. Provavelmente esta diferença esteja relacionada com o número de lâminas ou com a variabilidade das espécies estudadas especialmente em relação à densidade o que pode influenciar não apenas na mobilidade do adesivo como também na disposição das camadas externas e internas do painel. Portanto quanto maior o número de lâminas internas com fibras paralelas ao vão, mais uniforme será a resistência e a rigidez do compensado entre as duas direções.

\section{CONCLUSÕES}

Conforme os resultados desta pesquisa, as seguintes conclusōes podem ser apresentadas:

Os valores determinados na flexão estática indicam potencial de produção das espécies.

Entre as espécies, os painéis produzidos com $C$. duckei apresentaram valores médios superiores de módulo de elasticidade e módulo de ruptura em relação aos painéis de E. oleifera, embora o T1 do módulo de ruptura seja aproximadamente igual aos demais valores.

Os tratamentos aplicados nos painéis produzidos com E. oleifera não mostraram significância o que não interferiu aos valores de MOE e MOR podendo ser utilizado a menor gramatura. Quanto ao sentido perpendicular à grã dos corposde-prova o T2 foi o que obteve melhor resultado.

Os valores obtidos no ensaio de flexão estática tanto no sentido paralelo como perpendicular à grã dos corpos-de-prova podem ser considerados bons quando comparados com valores encontrados na literatura para painéis de outras espécies.

\section{BIBLIOGRAFIA CITADA}

Baldwin, R.F. 1795. Plywood Manufacturing Practies. San Francisco: Miller Freeman. 260pp.

Bodig, J.; Jayne, B. 1982. A Mechanics of wood and wood composites. New York: Van Nostrand Reinhold. 711pp.

European Committee for Standardization - European Standard EN 314-2. 1993. Plywood. Bonding quality. Part 2: Requirements. Bruxelas.

European Committee for Standardization - European Standard EN 310. 1993. Wood-based panels - Determination of modulus of elasticity in bending streng. Bruxelas.

Iwakiri, S.; Olandoski, D.P.; Leonhardt, G.; Brand, M.A. 2002. Produção de chapas de madeira compensada de cinco espécies de Pinus tropicais. Ciência Florestal, 11(2): 71-77. 
Kollmann, F.F.P.; Cotê, W.A. 1968. Prinples of wood science and technology. New York: Springer-Verlag, vol.1. 592pp.

Marra, A.A. 1992. Technology of wood bonding principles in practice. New York: Van Nostrand Reinhold. 453pp.

Pereyra, O. Avaliação da madeira de Eucalyptus dunnii (Maid) na manufatura de painéis compensados. 1994. Dissertação de Mestrado, Escola Superior de Agricultura Luís de Queiroz. Piracicaba, São Paulo. 87pp.

Selbo, M.L. 1975. Adhesive Bonding of wood. Technical Bulletin . $\mathrm{n}^{\circ}$ 1512. U. S. Department of Agriculture. Forest Service. Washington D.C. 122pp.
Sellers, T. 1985. Plywood and adhesive technology. New York: Marcel Dekker. 661pp.

Suchsland, O. 1972. Warping of furniture panels. Agric. Exp. Station, Michigan, Extension Bulletin E-745.

Tsoumis, G. 1991. Science and technology of wood - structure, properties, utilization. New York, Champman \& Hall. 494pp.

Recebido em 21/12/2006

Aceito em 05/07/2007 